\title{
Parâmetros de produtividade de um centro de material e esterilização*
}

\author{
PRODUCTIVITY INDICATORS INASTERILIZATION CENTRAL SUPPLY
}

LOS PARAMETROS DE PRODUCTIVIDAD DEL CENTRO DE MATERIAL Y ESTERILIZACIÓN

\author{
Tânia Regina Sancinetti ${ }^{1}$, Maria Alice Fortes Gatto²
}

\section{* Extraído da dissertação "Identificação de parâmetros de produtividade de um Centro de Material e Esterilização". Escola de Enfermagem da USP, 2002. \\ 1 Enfermeira. Mestre em Enfermagem pela Escola de Enfermagem da USP. Diretora da Divisão de Enfermagem de Pacientes Externos do Departamento de Enfermagem do Hospital Universitário da USP. \\ trsanci@yahoo.com.br \\ 2 Professora \\ Assistente do Departamento de Enfermagem Médico- Cirúrgica da Escola de Enfermagem da USP.}

\section{RESUMO}

O estudo identifica parâmetros de produtividade no Centro de Material e Esterilização, com base em documentos, observação e cronometragem do processamento de duas amostras: caixa de herniorrafia e pacote de curativo. A produção média mensal foi de 30.466,42 artigos, a capacidade de produção, por hora, foi de 10,3 artigos por funcionário. $\mathrm{O}$ tempo de processamento do curativo do expurgo ao armazenamento foi, em média, de 295 minutos; o processo manual utilizou 46 minutos e o automatizado, 88 minutos. O tempo da caixa de herniorrafia foi de 329 minutos; o processo manual utilizou 60 minutos e o automatizado, 98 minutos. O processamento manual do curativo utilizou 59,23\% horas produtivas no expurgo; $26,31 \%$ guarda e distribuição; $10,94 \%$ esterilização e 3,28\% preparo. Quanto à caixa de herniorrafia: no preparo $16,20 \%$; no expurgo $11,84 \%$; na guarda e distribuição 7,47\% e na esterilização 1,89\%. Este estudo possibilitou aferir resultados e analisar o processo de trabalho no CME.

\section{DESCRITORES}

Eficiência.

Administração de materiais no hospital.

Esterilização.

Enfermagem.

\section{ABSTRACT}

This study identifies productivity indicators at a Sterilization Central Supply (CME) through documents, observation and time counting of the process for two items selected from the sample: a bandage pack and a herniorrhaphy box. The monthly average production was $30,466.42$ items, and the production capacity per hour was 10.3 items per employee. The bandage pack averaged 295 minutes from the expurgation area to storage; the manual process lasted 46 minutes and the automated 88 minutes. For the herniorrhaphy box the average was 329 minutes; the manual process lasted 60 minutes and automated 98 minutes. In the bandage kit, the manual process used up $59.23 \%$ of the productivity hours in the expurgation area, $3.28 \%$ in the preparation, $10.94 \%$ in the sterilization process, and $26.31 \%$ in storage and distribution. Figures for the manual process of herniorrhaphy box were: expurgation, $11.84 \%$; preparation, $16.20 \%$; storage and distribution, $7.47 \%$; and sterilization, $1.89 \%$. The study made possible to assess the results and to analyze the working process at the CME.

\section{KEY WORDS}

Efficiency.

Materials management, hospital. Sterilization.

Nursing.

\section{RESUMEN}

El estudio identifica parámetros de productividad del Centro de Material y Esterilización, basados en documentos, observación y cronometraje procesadas en dos muestras: caja de herniorafia y fardo de curativo. El promedio de producción mensual fue de $30.466,42$ artículos y el promedio de la capacidad de producción por hora fue 10,3 artículos por empleado. El tiempo de procesamiento del curativo del expurgo al almacenamiento fue en un promedio de 295 minutos; su proceso manual utilizó 46 minutos y lo automatizó en 88 minutos. El tiempo de la caja de herniorafia fue 329 minutos; su proceso manual utilizó 60 minutos y lo automatizó en 98 minutos. El procesamiento manual del curativo fue: $59,23 \%$ horas productivas encima del expurgo; $26,31 \%$ guarda $\mathrm{y}$ distribución; $10,94 \%$ esterilización y $3,28 \%$ preparo. Cuanto a la caja de herniorafia: en su preparo $16,20 \%$; en el expurgo $11,84 \%$; en la custodia y asignación $7,47 \%$ y en la esterilización $1,89 \%$. El estudio posibilitó contrastar resultados y analizar el proceso de trabajo del CME.

\section{DESCRIPTORES}

Eficiencia.

Administración de materiales de hospital.

Esterilización. Enfermería. 


\section{INTRODUÇÃO}

A falta de instrumentos inviabiliza a análise dos recursos quantitativos e qualitativos das atividades desenvolvidas no Centro de Material e Esterilização (CME). A partir desta contastação, iniciou-se um processo de construção de mecanismos de controle e de análise de dados estatísticos desse setor e da adequação dos instrumentos a fim de que permitissem o entendimento e a mensuração do processo de trabalho do CME.

O CME tem finalidades e objetivos nitidamente definidos, tais como: concentrar os artigos odonto-médico-hospitalares, esterilizados ou não, facilitando seu controle, conservação e manutenção; padronizar e realizar técnicas de limpeza, preparo, empacotamento e esterilização, assegurando economia de pessoal, material e tempo; distribuir material esterilizado ou não aos diversos setores de atendimento a pacientes; treinar pessoal para atividades específicas do setor, conferindo-lhe maior produtividade; facilitar o controle de consumo, qualidade dos materiais e das técnicas de esterilização, aumentando a segurança do uso; favorecer o ensino e o desenvolvimento de pesquisas; manter estoque de material, a fim de atender prontamente à necessidade de qualquer unidade do hospital ${ }^{(1-2)}$.

As características de trabalho do CME são bastante distintas de outras Unidades do hospital gerenciadas pela enfermagem. Essa diferenciação ocorre, pela necessidade de aprofundar e aplicar os conhecimentos da área de microbiologia, da infecção hospitalar, dos processos de limpeza, desinfecção e esterilização de material. A segunda característica marcante do trabalho, visa manter um entrosamento mais efetivo com os setores usuários de seu serviço, tendo incorporado também conceitos relacionados à produtividade, qualidade e adequação às necessidades dos diversos setores do hospital ${ }^{(3-5)}$.

Nos últimos anos, verifica-se que a palavra produtividade tem aparecido com freqüência cada vez maior não só em revistas especializadas, mas na mídia em geral. Empresas preparam programas de melhoria da produtividade, simpósios e encontros são realizados, consultores especialistas são contratados. Produtividade tornou-se a palavra de ordem, uma espécie de "Abre-te Sézamo" para um sem-número de problemas da empresa particularmente lucro e sobrevivência ${ }^{(6)}$.

$\mathrm{Na}$ avaliação da produtividade é fundamental analisar o tempo despendido pelos trabalhadores na execução de suas tarefas. A medida do tempo recebeu destaque a partir de 1881, com os trabalhos do Engenheiro Frederich Taylor, em busca de uma produtividade justa e adequada de seus trabalhadores. O tempo foi medido com cronômetro e o trabalho dividido em fases, que medidas separadamente indicam o tempo mínimo de uma atividade ${ }^{(7-8)}$.
Na época o enfoque era o aumento da produção e do lucro independente do desgaste do material e prejuízo que traria ao trabalhador.

São raras as instituições de saúde no país que desenvolvem na prática a avaliação e o controle sistemático de suas atividades, passos fundamentais para a verificação e acompanhamento da produtividade e qualidade do serviço desenvolvido. $\mathrm{Na}$ área hospitalar, há necessidade do aumento da produtividade como forma de equilibrar os custos, em decorrência dos insuportáveis níveis elevados ${ }^{(9-11)}$

As medidas de tempo ganham outro significado a partir dos anos 80 com os estudos na área de avaliação da qualidade dos sistemas de saúde, desenvolvidos por Donabedian, o primeiro a propor métodos de avaliação de serviços de saúde e estratégias direcionadas à mensuração do alcance dos padrões estabelecidos para as áreas de estrutura, processo e resultado $^{(12)}$.

Mais recentemente, com a implantação do Sistema Brasileiro de Acreditação, procedimento de avaliação dos recursos institucionais, voluntário, periódico e reservado que tende a promover a qualidade da assistência por meio de padrões previamente aceitos e posteriormente mensurados e avaliados quanto ao alcance dos resultados e desempenho institucional, as instituições de saúde adquirem reconhecimento público e garantem a qualidade dos serviços prestados ${ }^{(13)}$.

No manual das organizações prestadoras de serviços hospitalares, o CME é referido como Serviços Assistenciais e de Abastecimento, responsável pelo processamento e esterilização de materiais e estabelece padrões de avaliação, classificados em três níveis. Entre as exigências para a inserção no nível 3, recomenda que "... participa ativamente do programa institucional de qualidade e produtividade, om evidências de ciclos de melhoria; está integrado ao sistema de informação da organização, utilização de dados e indicadores para avaliação..." ${ }^{(14)}$.

Não se pode deixar de considerar que o indicador deve contribuir para a administração do setor, e não servir como mais um dado complicado de se obter ou um trabalho desnecessário e repetitivo, tendo em vista apenas o preenchimento dos impressos existentes para avaliar a produção e distribuição de material destinado às diversas áreas do hospital ${ }^{(2)}$. $\mathrm{O}$ Indicador é um sinalizador que dirige a atenção para assuntos específicos de resultados em uma organização de saúde, devendo periodicamente ser revisto ${ }^{(15)}$.

O acompanhamento sistemático dos dados obtidos nos instrumentos de controle utilizados no CME e a análise periódica do tempo dos processos de trabalho podem se tornar parâmetros indicativos de produtividade.

O controle embora possa parecer o último passo de um processo, constitui apenas o elo de uma cadeia que alimentada continuadamente impulsiona as organizações para o 
desenvolvimento, pela melhoria de seus processos e resul$\operatorname{tados}^{(16)}$.

Dada à escassez de literatura sobre o assunto e na tentativa de evitar um mero exercício numérico ${ }^{(6)}$, este estudo propõe alguns parâmetros de mensuração dos processos de produção visando a melhoria dos resultados quanto à produtividade e qualidade no CME. Visa também contribuir com subsídios para o trabalho do enfermeiro no gerenciamento da unidade.

\section{OBJETIVOS}

- Identificar parâmetros de produtividade em um centro de material e esterilização de um hospital universitário da cidade de São Paulo;

- Identificar a frequiência mensal de processamento e distribuição dos artigos odonto-médico-hospitalares;

- Identificar o tempo de processo e o tempo de espera dos artigos odonto-médico-hospitalares submetidos ao processamento de limpeza, preparo, esterilização e estocagem realizados no $\mathrm{CME}$;

- Associar as medidas de tempo aos recursos materiais e de pessoal disponíveis nos setores do CME.

\section{MÉTODO}

\section{Tipo de pesquisa e campo de estudo}

O estudo caracterizou-se como não experimental, descritivo, exploratório e com abordagem quantitativa. Foi realizado no CME do Hospital Universitário da Universidade de São Paulo (HU-USP), que tem como objetivos: receber, conferir, selecionar, lavar, secar, preparar, identificar, esterilizar, armazenar, controlar e distribuir artigos odonto-médico-hospitalares que são utilizados pelas diversas unidades do hospital.

A escolha do campo de estudo foi motivada pelos vínculos existentes entre a Instituição e a pesquisadora, que na época atuava como enfermeira chefe do setor e, também, porque este CME é um setor estruturado, possui parte dos registros informatizados, de forma que os controles e rotinas existentes permitiram a verificação da produção das áreas e possibilitaram a realização do presente estudo.

\section{Objeto do estudo e técnica de amostragem}

O objeto deste estudo foi constituído pelos artigos odonto-médico-hospitalares processados no CME que são compostos por um ou mais itens (ou peças) e são embalados de modo unitário (pacote unitário) ou em conjunto (pacotes, conjuntos ou caixa de instrumental).

Para selecionar uma amostra, que representasse o trabalho executado em todos os setores do CME, foi estabelecido como critério a escolha de um artigo odonto-médico-hospitalar que tivesse a maior freqüência mensal de processamento e o maior esforço de trabalho, quer pelo número de itens que o compõe e, ou ainda, pelo maior número de tarefas realizadas nas etapas de limpeza, preparo, esterilização e guarda.

Foram selecionados dois artigos como amostras para este estudo: o pacote de curativo cirúrgico e a caixa de herniorrafia.

\section{Coleta de dados}

\section{Fontes}

- Documentos de controle da instituição: relatório mensal, quadro de pessoal, escala mensal, escala de distribuição diária de trabalho, manuais do CME e impressos de previsão e produção dos meses de agosto de 2000 a julho de 2001;

- Descrição de observação das tarefas e da trajetória do processamento das amostras.

\section{Procedimentos}

O projeto foi submetido e aprovado pelo Comitê de Ensino e Pesquisa (CEP) da Instituição.

Após a autorização formal da instituição para a realização da pesquisa foi agendada uma reunião com os funcionários do CME, para explicar os objetivos do presente estudo, esclarecer que os procedimentos da coleta de dados não interfeririam nos processos de trabalho, nem na dinâmica do setor e que os resultados seriam apresentados ao grupo no final do estudo.

Foi explicitada a participação voluntária e solicitada à assinatura do Termo de Consentimento Livre e Esclarecido. A coleta de dados ocorreu em três etapas:

$1^{\text {a }}$ etapa: seleção e levantamento dos dados documentais;

$2^{a}$ etapa: classificação dos artigos;

$3^{\text {a }}$ etapa: acompanhamento das amostras.

Todas as atividades, realizadas com cada um dos artigos selecionados como amostras, foram observadas durante dez processamentos, desde o momento da recepção no expurgo até a estocagem na área de guarda e distribuição. 


\section{APRESENTAÇÃOE DISCUSSÃO DOS RESULTADOS}

\section{Produção e distribuição dos artigos}

A freqüência do processamento (dados de produção) foi obtida do registro da produção das áreas de preparo do CME. O total de artigos produzidos no período estudado (agosto de 2000 a julho de 2001) foi 365.597 artigos, com uma média mensal de 30.466,42 artigos.

Os dados de distribuição corresponderam aos artigos encaminhados pelo CME às unidades usuárias, estes dados estão expressos no impresso Troca de Material.
O total dos artigos distribuídos às unidades consumidoras, no período estudado, foi 361.232 artigos sendo a média de distribuição: 30103 artigos/mês.

Os dados apresentados na Figura 1, demonstram que os meses de dezembro de 2000, fevereiro e julho de 2001, a produção do CME foi menor $(27.815,25.831,27.269$ respectivamente); os meses de agosto, abril e maio são os de maior produção $(35.065,34.477,33.856$ respectivamente). Nota-se que a diferença entre as maiores e menores produções ocorreu em torno de $22,2 \%$.

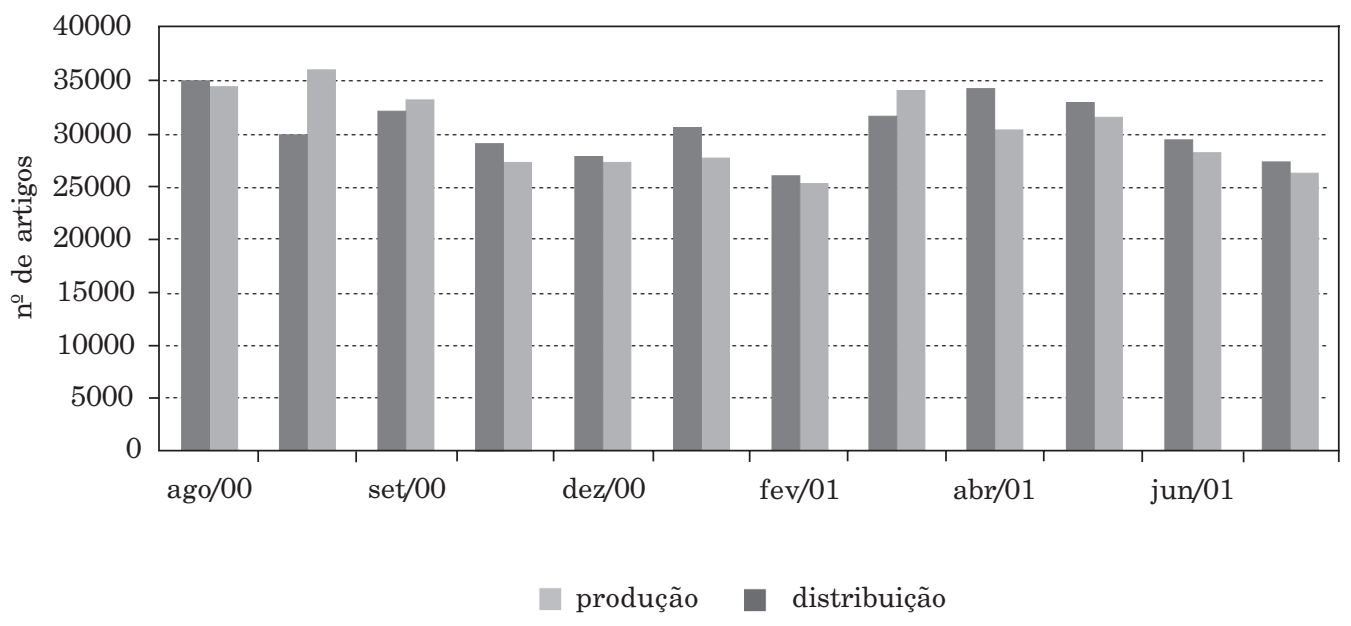

Figura 1 - Apresentação do total da produção e distribuição dos artigos do CME / mês, HU-USP - São Paulo - 2001

Pode-se verificar que, no mês de setembro, a distribuição foi maior que a produção e nesse mês, encontrou-se a maior ausência de funcionários, que justificou a queda na produção.

Também nos meses de outubro/00 e março/01 notou-se diferença entre distribuição e produção, que foram compensadas com estoques de material na área de guarda.

No mês de fevereiro, a queda tanto da produção quanto da distribuição, que pôde ser relacionada ao menor número de dias trabalhados.

Verificou-se uniformidade entre produção e distribuição; as variações encontradas demonstram que a produção está adequada e não restam estoques desnecessários.

Relacionando os dados de produção (Figura 1) com a freqüência de funcionários (Tabela 1), verificou-se que nas férias de verão a capacidade de horas de pessoal, também estava reduzida, porém no mês de julho, a produção diminuiu, no entanto a capacidade de pessoal estava aumentada. Dessa forma, é possível propor que para otimizar o tempo do funcionário, essa época seja utilizada, por exemplo, para visitas técnicas e treinamentos.

\section{Produção por hora/funcionário}

Biseng apresenta níveis de produtividade entre $60 \%$ a $85 \%$ e os avalia como: insatisfatório até $60 \%$; satisfatório, entre $60 \%$ e $75 \%$; excelente quando maior de $75 \%$ a $85 \%$ e suspeito se for maior que $85 \%{ }^{(17)}$.

Para estabelecer uma relação entre produção dos artigos e a capacidade de horas de trabalho, no período de agosto de 2000 a julho de 2001, utilizamos no estudo, $75 \%$ do tempo do trabalho, como nível de produtividade aceitável. 
Tabela 1 - Demonstrativo da produção por horas/funcionário de enfermagem por mês, HU-USP - São Paulo - 2001

\begin{tabular}{cccrc}
\hline MESES & $\begin{array}{c}\text { TOTAL } \\
\text { PRODUÇÃO }\end{array}$ & $\begin{array}{c}\text { TOTAL } \\
\text { HORAS/FUNC }\end{array}$ & $\begin{array}{r}\text { TOTAL } \\
\text { PRODUTIVAS }\end{array}$ & $\begin{array}{c}\text { PRODUÇÃO } \\
\text { HORA }\end{array}$ \\
\hline ago/00 & 35.065 & 4.074 & $3.055,5$ & 11,5 \\
set/00 & 29.818 & 3.750 & $2.812,5$ & 10,6 \\
out/00 & 32.017 & 3.696 & 2.772 & 11,6 \\
nov/00 & 29.072 & 3.750 & $2.812,5$ & 10,3 \\
dez/00 & 27.815 & 3.954 & $2.965,5$ & 9,4 \\
jan/01 & 30.485 & 3.864 & 2.898 & 10,5 \\
fev/01 & 25.831 & 3.690 & $2.767,5$ & 9,3 \\
mar/01 & 31.608 & 3.828 & 2.871 & 11,0 \\
abr/01 & 34.474 & $3.974,5$ & 11,6 \\
mai/01 & 32.859 & 3.966 & 9.339 & 9,8 \\
jun/01 & 29.284 & 4.452 & $3.154,5$ & 8,3 \\
\hline jul/01 & 27.269 & 4.206 & $3.055,5$ & 8,9 \\
\hline Total & 365.597 & 4.074 & 35.478 & 123,8 \\
\hline Média & 30466,4 & 47.298 & $2.956,5$ & 10,3 \\
\hline DP & $2.846,7$ & $3.941,5$ & 172,5 & 1,0 \\
\hline
\end{tabular}

No período (agosto 00/julho 01), a produção total foi de 365.597 artigos (30.466,4 média mensal) e a frequiência total dos funcionários foi 47.298 horas (média 3.941,5). Considerando $75 \%$ do tempo da freqüência, a média mensal das horas produtivas foi de 2.956,5 horas. Encontrou-se que cada funcionário poderia participar do processamento de, em média, 10,3 artigos por hora (no mínimo 8,9 artigos e máximo 11,6 artigos).

\section{Tempo de processamento dos artigos selecionados como amostras}

Nesta fase do estudo, procurou-se conhecer os tempos percorridos pelas amostras em seu processamento.
O pacote de curativo e a caixa de herniorrafia foram acompanhados desde sua entrada no CME pelo setor de expurgo, até sua estocagem no setor de guarda e distribuição.

O tempo médio que o pacote de curativo cirúrgico percorreu no CME, desde a sua entrada no expurgo até o armazenamento na área de guarda de distribuição foi no total 295 minutos (4:55 horas), o tempo mínimo foi 217 minutos e o máximo 394 minutos (Figura 2).

Quanto à caixa de herniorrafia, o tempo médio foi 329 minutos no total, sendo o tempo mínimo de 221 minutos e o máximo de 454 minutos (Figura 2).

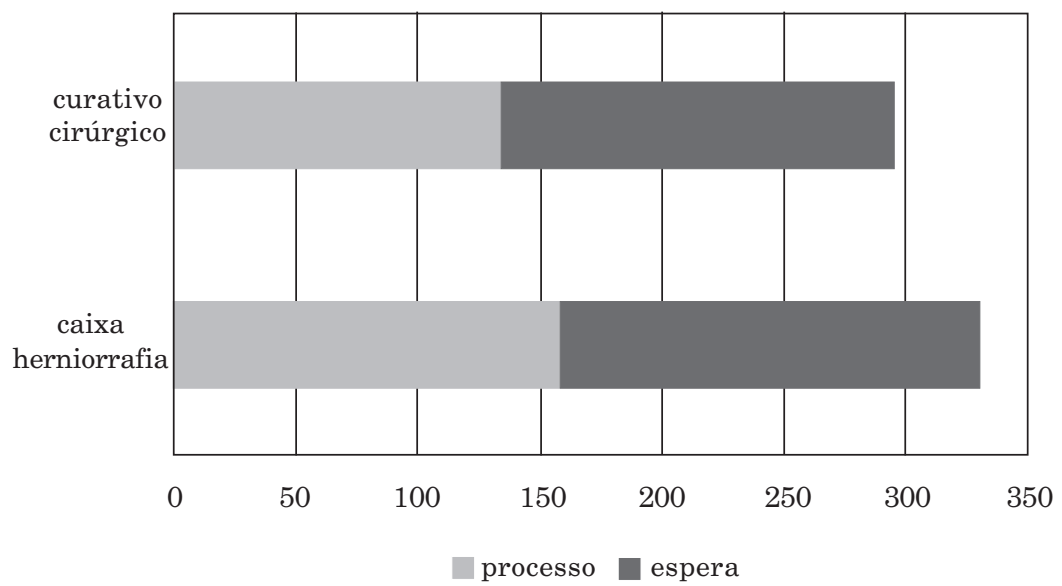

Figura 2 - Tempo médio em minutos do processo de trabalho das amostras, em procedimento e em espera, HU-USP São Paulo - 2001 
Verificou-se que dos 295 minutos da trajetória do curativo cirúrgico, $45,42 \%$ do tempo aconteceu em procedimento e $54,67 \%$ do tempo, em espera.

Em relação à caixa de herniorrafia, dos 329 minutos da trajetória, $48,0 \%$ do tempo o artigo permaneceu em proce- dimento e $52,0 \%$ do tempo, em espera.

Estes tempos indicam que as amostras estão ficando paradas por um tempo superior à metade do processamento, indicando necessidade de observação do processo de trabalho.

Tabela 2 - Tempo médio em minutos das amostras em processo de trabalho (procedimento e espera), CME HU-USP São Paulo - 2001

CURATIVO CIRÚRGICO

AMOSTRA

TOTAL DO PROCESSO
CAIXA DE HERNIORRAFIA

TOTAL DO PROCESSO

\begin{tabular}{lcccccc} 
& TOTAL & PROCED & ESPERA & TOTAL & PROCED & ESPERA \\
\hline Média & 295 & 134 & 161 & 329 & 158 & 172 \\
\hline DP & 57 & 13 & 52 & 81 & 27 & 67 \\
\hline
\end{tabular}

Os curativos são parte de um grupo de artigos recebido pelo CME dos diversos setores usuários, como "troca de material". Dessa forma, contou-se o tempo de recebimento e de ordenação e guarda, do grupo como um todo. O tempo de processo das duas amostras, conforme tabela 2, são semelhantes.

Os motivos encontrados nos tempos maiores de espera foram: funcionário novo na área de preparo; dia de limpeza na área de guarda; prioridade para outras caixas; comemorações; passagem de plantão; montagem de carros cirúrgicos e caixas misturadas.

Observou-se grande variação nos tempos de espera e considerando-se que este é um processo produtivo, o tempo de espera deveria apresentar valores mais próximos e ficar restrito a um tempo mínimo.

Tabela 3 - Tempo médio de trabalho em minutos das amostras em procedimento automatizado e manual nos diversos setores do CME HU-USP - São Paulo - 2001

\begin{tabular}{lccccc} 
& \multicolumn{2}{c}{ EXPURGO } & PREPARO & ESTERILIZAÇÃO & $\begin{array}{c}\text { GUARDA E } \\
\text { DIST. }\end{array}$ \\
\cline { 2 - 6 } & Manual Automatizado & Manual & Manual Automatizado & Manual \\
\hline $\begin{array}{l}\text { Curativo } \\
\text { Cirúrgico }\end{array}$ & 27 & 45 & 2 & 5 & 43 \\
\hline $\begin{array}{l}\text { Caixa } \\
\text { Herniorrafia }\end{array}$ & 19 & 52 & 26 & 3 & 46 \\
\hline
\end{tabular}

O preparo da caixa é intensamente manual e, por ser artigo composto por vários itens, o processo tornou-se mais longo que o da amostra curativo cirúrgico. No expurgo, mesmo tendo sido utilizado processos manuais e automatizados, os tempos das duas amostras são semelhantes, devido à manipulação de cada um dos itens da caixa e dos diversos itens da troca de materiais a qual pertence o curativo cirúrgico. O setor de esterilização é automatizado, sendo o tempo de processo pré-estabelecido, assim como no processo automatizado do expurgo, existindo portanto pouca variação entre os tempos das duas amostras no que se refere a parte automatizada dos processos.

Tempo de processamento de artigos e disponibilidade de pessoal

Considerando a média mensal da produção de artigos do grupo curativo (3.891 artigos) o tempo em processo manual e a disponibilidade de funcionário em horas produtivas $(2.956 \mathrm{~h})$ encontrou-se que o processo desse artigo em um mês utilizou:

- EXPURGO: 1.751 horas/mês (59,23\%); 
- PREPARO: 130 horas/mês (3,28\%);

- ESTERILIZAÇÃO: 324 horas/mês (10,94\%);

- GUARDAEDISTRIBUIÇÃO: 778 horas/mês(26,31\%).

O expurgo e a guarda e distribuição são áreas reconhecidas pelos funcionários como desgastante, que necessita de investimentos para melhoria das condições de trabalho.

Associando os dados do grupo de caixas, sendo a média de produção de 1.106 caixas, verificou-se:

- EXPURGO:350horas/mês(11,84\%);

- PREPARO: 479 horas/mês (16,20\%);

- ESTERILIZAÇÃO: 56 horas/mês (1,89\%);

- GUARDAEDISTRIBUIÇÃO: 221 horas/mês(7,47\%).

Além da característica citada sobre o expurgo e a guarda e distribuição, também a área de preparo de caixas, por ser a mais utilizada nesta amostra em carga horária, deve ter condições favoráveis de trabalho.

A área de esterilização, apesar de ser mais automatizada, necessita de atuação e vigilância constantes, a fim de garantir a qualidade dos procedimentos realizados na Instituição.

\section{REFERÊNCIAS}

1. Moura MLPA. Gerenciamento da Central de Material e Esterilização para enfermeiros: fundamentos teóricos, organizacionais e estruturais. São Paulo: SENAC; 1996.

2. Silva A. Organização do trabalho na Unidade Centro de Material. Rev Esc Enferm USP. 1998; 32(2):169-78.

3. Gatto MAF. Análise da utilização de salas de operações [tese] São Paulo: Programa Interunidades, Escola de Enfermagem, Escola de Enfermagem de Ribeirão Preto, Universidade de São Paulo; 1996.

4. Antunes AV, Trevisan MA. Gerenciamento de qualidade: utilização no serviço de enfermagem. Rev Lat Am Enferm. 2002;8(2):35-44.

5. Tipple AFV, Pereira MS, Hayashida M, Moriya TM, Souza ACS. O ensino do controle de infecção: um enfoque teóricoprático. Rev Lat Am Enferm. 2003;11(2):245-50.

6. Moreira DA. Administração da produção e operações. São Paulo: Pioneira; 1993.

7. Barnes RM. Estudo de movimentos e de tempos: projeto e medida do trabalho. $6^{\text {a }}$ ed. São Paulo: Edgard Blücher; 1977. Definição e finalidades do estudo de tempos e de movimentos; p. 1-7.

8. Schoeps W. Estudo dos tempos e movimentos. In: Machiline C, Motta IS, Schoeps W, Weil KE, editors. Manual de administração da produção. $9^{\text {a }}$ ed. Rio de Janeiro: Fundação Getúlio Vargas; 1990. p. $480-523$.

\section{CONCLUSÕES}

A partir da análise e adequação dos instrumentos de trabalho, foi possível aferir os resultados da produção e distribuição dos artigos odonto-médico-hospitalares. A média mensal de produção (30.466,4 artigos) tem uma seqüência de processamento e distribuição uniformes, demonstrando adequação da previsão; a média de artigos produzidos por hora funcionário foi de 10,3; o tempo médio de processamento da amostra curativo cirúrgico foi de 295 minutos sendo $45,42 \%$ em procedimento e $54,67 \%$ em espera e da caixa de herniorrafia foi de 329 minutos, sendo $48 \%$ em procedimento e $52 \%$ em espera.

O processo de trabalho precisa ser melhor investigado, devido ao tempo de espera, que precisa ser restrito a um tempo mínimo. A espera observada também sugere necessidade de automação do processo, principalmente na área de expurgo.

As medidas de tempo foram, neste primeiro estudo, apenas somadas e não foram vinculadas à qualidade.

Considera-se necessário uma reflexão sobre "Até que ponto a medida física da produção é representativa do serviço! Até que ponto uma única medida consegue refletir a complexidade do conjunto de tarefas que se deve cumprir para realizar o serviço? ${ }^{(13)}$. Este é o desafio que espera-se seja ultrapassado com estudos complementares sobre a qualidade e produtividade emCME.

9. Beyers M. About productivity indicators. Nurs Manage. 1997;28(11):96.

10. Martinelli MCS. Estudo evolutivo de indicadores hospitalares do Hospital Ana Costa. Qualimetria. 1993;5(27):4-16.

11. Ide P, Kirby KK, Starck MS. Operating room productivity: an evaluation format. J Nurs Adm. 1992;22(10):41-8.

12. Donabedian A. The definition of quality and approaches to iits assessment. Ann Arbor, Mich: Health Administration Press; c1980. (His Explorations in Quality Assessment and Monitoring, v. 1).

13. Novaes HM, Paganini JM. Garantia de qualidade: acreditação de hospitais para América Latina e o Caribe. Genebra: OPAS/ OMS; 1992. (Série Silos, n. 13).

14. Organização Nacional de Acreditação. Manual das organizações prestadoras de serviços hospitalares; 2001. Brasília; 2001.

15. Bittar OJN. Indicadores de qualidade e quantidade em saúde. Rev Adm Saúde. 2001;3(12):21-8.

16. Silva SH. Controle de qualidade na assistência de enfermagem: implementação de um modelo. [tese]. São Paulo: Escola de Enfermagem, Universidade de São Paulo; 1994.

17. Gaidzinski RR. Dimensionamento de pessoal de enfermagem em instituições hospitalares [tese]. São Paulo: Escola de Enfermagem, Universidade de São Paulo; 1998. 